\title{
« Choisir avec soin » et la gestion des antimicrobiens : un même souci de réduire les soins inutiles
}

\author{
Born $\mathrm{KB}^{1^{*}}$, Leis $\mathrm{JA}^{2}$, Gold $\mathrm{WL}^{3}$, Levinson $\mathrm{W}^{1}$ \\ ${ }^{1}$ Choisir avec soin, Université de Toronto, Toronto (Ontario) \\ 2 Division des maladies infectieuses et Centre pour l'amélioration de la qualité et la sécurité des patients, Université de \\ Toronto, Toronto (Ontario) \\ ${ }^{3}$ Division des maladies infectieuses, Université de Toronto, Toronto (Ontario) \\ * Correspondance : karen.born@mail.utoronto.ca
}

\section{Résumé}

On constate dans le milieu médical un mouvement croissant qui reconnaît que certains examens, certains traitements ou certaines interventions médicales n'ajoutent aucune valeur pour les patients, et peuvent même être nocifs. La campagne « Choisir avec soin » est une campagne populaire dirigée par des médecins, qui vise à engager le dialogue entre les médecins et les patients sur l'utilisation excessive d'examens, de traitements et d'interventions médicales inutiles, et à améliorer la qualité des soins de santé. Cet article examine les principes sous-jacents de cette campagne et sa progression dans le pays. Il met également en évidence I'harmonisation entre les principes de "Choisir avec soin » et ceux de la gestion des antimicrobiens, qui ont en commun des motivations, des difficultés et des possibilités similaires.

\section{Introduction}

L'amélioration de la qualité, de la sécurité et des soins axés sur les patients est l'objectif commun des prestataires, des organisations et des systèmes de soins de santé dans tout le Canada. Les études canadiennes et internationales ont révélé l'ubiquité des événements indésirables et des préjudices associés aux soins médicaux $(1,2)$. Le mouvement pour la sécurité des patients a souligné qu'une partie de ces préjudices est évitable, et a mis en œuvre diverses stratégies comme des listes de vérification, des ensembles, des mesures améliorées et des programmes de perfectionnement professionnel continu. L'un des objectifs importants du mouvement pour la sécurité des patients est la prévention des soins inutiles, que l'on définit comme les soins médicaux prodigués aux patients qui n'offrent aucun bienfait ou n'ont pas d'indication clinique. Lorsque les soins n'apportent aucun bienfait ou ne sont pas cliniquement indiqués, l'objectif d'amélioration de la qualité devient luimême des soins inutiles. Les soins inutiles sont motivés par plusieurs forces complexes, comme les habitudes de pratique établies, les contraintes de temps, le malaise associé à l'incertitude du diagnostic et les attentes perçues des patients.

Même s'ils sont potentiellement bien intentionnés, les soins inutiles peuvent déclencher, par " effet de cascade ", d'autres examens, interventions médicales et traitements qui peuvent causer des préjudices physiques et psychologiques (3). Des recherches plus poussées peuvent mener à des événements indésirables, à des complications, ainsi qu'à une augmentation du stress et de l'anxiété chez le patient. Les soins inutiles peuvent également grever des budgets en soins de santé déjà serrés. Selon des données provenant des États-Unis, $30 \%$ des dépenses en soins de santé sont excessives (4). Même s'il n'existe aucun chiffre global similaire au Canada, de plus en plus de documents font état de l'omniprésence des examens, traitements et interventions médicales inutiles (5).

Même si les médecins et les patients s'accordent pour dire que l'amélioration de la qualité des soins et la réduction de l'utilisation excessive des examens, traitements et interventions médicales inutiles sont un objectif important, la modification du comportement des médecins et des attentes des patients est difficile et complexe. 
La campagne "Choisir avec soin » vise à aborder ces causes complexes en fournissant des documents éducatifs fondés sur des données probantes aux médecins et aux patients, en encourageant la conversion sur les choix éclairés et en facilitant un dialogue public plus élargi au sujet des soins inutiles. Cet article a pour objectif de passer en revue les principes sous-jacents de cette campagne et de mettre en lumière l'harmonisation entre les principes de « Choisir avec soin » et ceux de la gestion des antimicrobiens.

\section{"Choisir avec soin » : Une campagne dirigée par les médecins}

La campagne "Choisir avec soin » a été lancée en avril 2014. Elle s'inspire de la campagne "Choosing Wisely » de l'American Board of Internal Medicine Foundation lancée en avril 2012, qui encourage les médecins et les patients à parler des examens, traitements et interventions médicales inutiles afin d'améliorer la valeur des soins et de réduire les préjudices.

À ce jour, 45 sociétés canadiennes de spécialistes se sont jointes à la campagne. Les sociétés participantes s'engagent à élaborer une « liste des 5 principaux examens et traitements que les médecins et les patients devraient remettre en question ». Ces recommandations énoncent ce que les médecins devraient cesser de faire; à ce jour, il en existe plus de 100, et d'autres recommandations sont sur le point d'être publiées.

\section{« En matière de soins, plus n'est pas toujours mieux »}

Les recommandations et les listes de " Choisir avec soin » visent à stimuler le dialogue entre les patients et les médecins afin qu'ils déterminent ensemble un parcours de soins et un plan de traitement. Chaque liste contient les examens, les traitements et les interventions médicales du champ d'exercice d'une spécialité pour lesquels il existe d'excellentes preuves d'utilisation excessive, de gaspillage ou de préjudice pour les patients. Ces listes sont accessibles au public sur le site Web de « Choisir avec soin ».

Les sociétés de spécialistes ont utilisé divers procédés pour dresser les listes, mais en suivant les principes ciaprès :

1. Le processus d'élaboration est bien documenté et accessible au public.

2. Chaque recommandation est dans le champ d'exercice de la spécialité.

3. Les examens, traitements et interventions médicales qui en font partie sont a) fréquemment utilisés et b) peuvent exposer les patients à un préjudice ou à un stress.

4. Chaque recommandation est appuyée par des preuves.

On constate la présence d'un mouvement international croissant autour de cette approche; des programmes "Choisir avec soin » sont lancés dans quinze pays. Un groupe de travail international, dirigé par le programme "Choisir avec soin » du Canada, a formulé un ensemble de cinq principes qui doivent être incorporés dans une campagne "Choisir avec soin »: elle doit être dirigée par les médecins, axée sur les patients, fondées sur les données probantes, multiprofessionnelle et transparente (6).

\section{La gestion des antimicrobiens et « Choisir avec soin »}

La prise en charge des syndromes de maladies infectieuses est souvent empirique; le traitement est instauré en l'absence de preuves complètes et repose sur une décision clinique raisonnée. Dans certains cas, les résultats des examens peuvent être en attente au moment de l'instauration du traitement antimicrobien; dans d'autres, les résultats peuvent être négatifs, alors qu'une infection est bel et bien présente. Le choix judicieux des examens diagnostiques, y compris les situations dans lesquelles il convient de ne pas effectuer d'examens, est un principe 
important commun à « Choisir avec soin » et à la prise en charge des maladies infectieuses, afin d'assurer une prescription rationnelle et sécuritaire des traitements antimicrobiens (7).

Au-delà de la prise de décisions judicieuses concernant les examens, d'autres principes de la gestion des ressources intéressent les spécialistes en maladies infectieuses depuis longtemps (8). À une époque d'augmentation de la résistance aux antimicrobiens et de choix limité de nouveaux agents antimicrobiens efficaces, les programmes de gestion des antimicrobiens sont devenus une composante fondamentale des systèmes de soins de santé au Canada. Les programmes de gestion des antimicrobiens sont l'une des façons dont les organisations et les médecins mettent en pratique les motivations qui sous-tendent " Choisir avec soin ».

Les programmes de gestion des antimicrobiens sont récemment devenus une pratique organisationnelle obligatoire d'Agrément Canada. Les données portent à croire que, pour qu'un programme de gestion des antimicrobiens soit efficace, des stratégies locales doivent être en place pour veiller à ce que ce programme désormais obligatoire soit accepté de la part des médecins de première ligne (9). En revanche, « Choisir avec soin » est fondamentalement une campagne populaire; néanmoins, elle s'harmonise étroitement avec les principes et les objectifs des programmes de gestion des antimicrobiens. Les deux programmes visent à optimiser les résultats pour les patients tout en réduisant au minimum les préjudices involontaires associés à l'utilisation des agents antimicrobiens, et ont l'objectif secondaire de réduire les coûts des soins de santé sans toucher à la qualité.

Les programmes de gestion des antimicrobiens font face à des défis semblables dans le domaine du changement de l'exercice de la médecine et des attentes des patients. II existe une documentation abondante concernant les interventions de gestion des antimicrobiens et leurs répercussions sur l'amélioration de l'utilisation des agents antimicrobiens, la réduction de la résistance aux antimicrobiens et l'amélioration des résultats cliniques $(10,11)$. Malgré cela, l'utilisation excessive des agents antimicrobiens et les effets indésirables qui y sont associés restent un problème courant important. La prescription des agents antimicrobiens est un comportement influencé par de nombreux déterminants sociaux et culturels, ainsi que par des comportements individuels enracinés qui ont généralement été motivés par les attentes perçues des patients et d'autres facteurs (12).

"Choisir avec soin » offre une autre tribune qui facilite le dialogue entre les médecins et les patients concernant les pratiques de prescription des agents antimicrobiens. Le tableau 1 ci-dessous montre une sélection de recommandations tirées des listes des sociétés de spécialistes canadiennes et américaines concernant l'utilisation inutile ou inappropriée des agents antimicrobiens selon l'angle de la pratique clinique. Bon nombre de ces exemples d'utilisation excessive d'agents antimicrobiens ont trait aux traitements ambulatoires, où la gestion des antimicrobiens a traditionnellement été plus difficile à mettre en œuvre (13). Les recommandations de "Choisir avec soin » axées sur l'utilisation des antibiotiques donnent l'occasion d'encourager le dialogue général sur l'utilisation appropriée des agents antimicrobiens, au-delà de ce qui peut être abordé dans les programmes de gestion des antimicrobiens en milieu hospitalier.

Tableau 1 : Exemples de recommandations "Choisir avec soin " des sociétés de spécialistes canadiennes et américaines sur les agents antimicrobiens

\begin{tabular}{|c|c|}
\hline Groupes & Recommandations \\
\hline \multicolumn{2}{|r|}{ Société de spécialistes canadienne } \\
\hline $\begin{array}{l}\text { Société canadienne de } \\
\text { gériatrie }\end{array}$ & $\begin{array}{l}\text { N'utilisez pas d'antimicrobiens pour traiter la bactériurie chez les } \\
\text { personnes âgées à moins d'être en présence de symptômes particuliers } \\
\text { d'infection urinaire. }\end{array}$ \\
\hline
\end{tabular}




\begin{tabular}{|l|l|}
\begin{tabular}{|l|l|}
$\begin{array}{l}\text { Le Collège des médecins de } \\
\text { famille du Canada }\end{array}$ & $\begin{array}{l}\text { N'utilisez pas d'antibiotiques pour traiter une infection des voies } \\
\text { respiratoires supérieures vraisemblablement d'origine virale, comme une } \\
\text { maladie s'apparentant à la grippe, ou spontanément résolutive, comme } \\
\text { une infection des sinus qui dure depuis moins de sept jours. }\end{array}$ \\
\hline \multicolumn{1}{|c|}{ Groupes } & \multicolumn{1}{c|}{ Recommandations } \\
Canada
\end{tabular} & $\begin{array}{l}\text { Éviter de prescrire un agent antimicrobien pour traiter une bactériurie } \\
\text { asymptomatique chez l'homme âgé. }\end{array}$ \\
\hline \multicolumn{1}{|c|}{ Société de spécialistes américaine } \\
\hline $\begin{array}{l}\text { American Academy of } \\
\text { Dermatology }\end{array}$ & $\begin{array}{l}\text { Éviter de prescrire un traitement antifongique par voie orale pour une } \\
\text { infection fongique des ongles soupçonnée, mais non confirmée. }\end{array}$ \\
\hline $\begin{array}{l}\text { American Academy of } \\
\text { Dermatology }\end{array}$ & $\begin{array}{l}\text { Éviter d'utiliser des antibiotiques par voie orale pour le traitement de la } \\
\text { dermatite atopique, sauf en présence de manifestations cliniques } \\
\text { d'infection. }\end{array}$ \\
\hline $\begin{array}{l}\text { American Academy of Family } \\
\text { Physicians }\end{array}$ & $\begin{array}{l}\text { Éviter de prescrire systématiquement des antibiotiques pour une sinusite } \\
\text { aiguë légère à modérée, à moins que les symptômes persistent pendant } \\
\text { au moins sept jours ou empirent après une amélioration clinique initiale. }\end{array}$ \\
\hline $\begin{array}{l}\text { American Academy of } \\
\text { Ophthalmology }\end{array}$ & $\begin{array}{l}\text { Éviter de prescrire des antibiotiques pour une conjonctivite à adénovirus } \\
\text { (conjonctivite à bacille de Weeks). }\end{array}$ \\
\hline
\end{tabular}

\section{Difficultés et possibilités communes}

"Choisir avec soin » et les programmes de gestion des antimicrobiens ont en commun bon nombre de motivations et de défis. Dans le contexte de ressources en soins de santé limitées, les deux initiatives peuvent être conçues par certains critiques comme des exercices de réduction des coûts ou de rationnement, plutôt que comme des efforts d'amélioration de la qualité des soins (3). La campagne « Choisir avec soin » au Canada et à l'échelle internationale reste indépendante du gouvernement; il est évident pour tous les intervenants que les recommandations ne doivent pas être utilisées par les décideurs pour radier des services de la liste ou déterminer lesquels sont payés. En fait, une telle radiation des listes serait difficile à mettre en œuvre, puisque les recommandations n'interdisent pas les agents antimicrobiens dans toutes les circonstances et reposent sur la prise de décisions cliniques. En outre, certains problèmes d'évaluation sont associés aux deux efforts, car il peut être difficile de quantifier les préjudices évitables ou les soins « appropriés » prodigués. Les données sont essentielles pour obtenir le soutien des médecins, des bailleurs de fonds et des intervenants pour ces initiatives; des efforts sont en cours pour assurer une meilleure évaluation et montrer les répercussions de la modération sur les résultats pour les patients.

Sous l'angle des possibilités, les principes de « Choisir avec soin » et les programmes de gestion des antimicrobiens sont étroitement harmonisés. De même, il existe de nombreux leviers du changement pour ces efforts, qui sont des initiatives dirigées par les médecins visant à modifier la pratique, à éviter les préjudices et à améliorer la gestion de ressources précieuses en soins de santé. Ces efforts peuvent à leur tour influencer les attentes du public et des patients et contribuer à favoriser le dialogue afin de faire des choix intelligents et efficaces qui assurent une qualité de soins élevée.

\section{Conflit d'intérêts}

Aucun 


\section{Financement}

Aucun

\section{Références}

(1) Brennan TA, Leape LL, Laird NM, Hebert L, et al. Incidence of adverse events and negligence in hospitalized patients-Results of the Harvard Medical Practice Study. N Engl J Med. 1991;324:370-6.

(2) Baker GR, Norton PG, Flintoft V, et al. The Canadian Adverse Events Study: The incidence of adverse events among hospital patients in Canada. CMAJ. 2004;170(11):1678-86.

(3) Deyo R. Cascade effects of medical technology. Ann Rev Pub Health. 2002;23:22-44.

(4) Institute of Medicine (US) Roundtable on Evidence-Based Medicine. Yong PL, Saunders RS, Olsen LA, editors. The Healthcare Imperative: Lowering Costs and Improving Outcomes-Workshop Series Summary. Washington: National Academies Press (US); 2010. p. 1-9.

(5) Emery DJ, et al. Overuse of magnetic resonance imaging. JAMA Intern Med. 2013;173(9):823-5.

(6) Levinson W, Kallewaard M, Bhatia RS, et al. 'Choosing Wisely': A growing international campaign. BMJ Qual Saf. 2014 Dec 31. doi:10.1136/bmjqs-2014-003821.

(7) Mandell, LA, Wunderink RG, Anzueto A, et al. Infectious Diseases Society of America/American Thoracic Society Consensus Guidelines on the Management of Community-Acquired Pneumonia in Adults. Clin Infect Dis. 2007;44:S27-71.

(8) Dellit T, Owens R, McGowan J, et al. Infectious Diseases Society of America and the Society for Healthcare Epidemiology of America Guidelines for Developing an Institutional Program to Enhance Antimicrobial Stewardship. Clin Infect Dis. 2006;44:159-77.

(9) Bal AM, Gould IM. Antibiotic stewardship: Overcoming implementation barriers. Curr Opin Infect Dis. 2011;24(4):357-62.

(10) Cosgrove SE, Seo SK, Bolon MK, et al. Evaluation of postprescription review and feedback as a method of promoting rational antimicrobial use: A multicenter intervention. Infect Control Hosp Epidemiol. 2012;33(4):374-80.

(11) Elligsen M, Walker SA, Pinto R, Simor A, et al. Audit and feedback to reduce broad-spectrum antibiotic use among intensive care unit patients: A controlled interrupted time series analysis. Infect Control Hosp Epidemiol. 2012;33(4):354-61.

(12) Charani E, Castro-Sanchez E, Sevdalis N, Kyratsis Y, et al. Understanding the determinants of antimicrobial prescribing within hospitals: The role of "prescribing etiquette." Clin Infect Dis. 2013;57(2):188-96.

(13) Drekonia DM, Filice GA, Greer N, et al. Antimicrobial stewardship in outpatient settings: A systematic review. Infect Control Hosp Epidemiol. 2015;36(2):142-52. 